DOI 10.31489/2021No3/88-92

UDC 536.2

\title{
MODEL OF VOLUMETRIC HEAT SOURCES IN ANODE OF MICROFOCUS X-RAY TUBE
}

\author{
Kozlov E.A., Trubitsyn A.A., Fefelov A.A., Grachev E.Yu. \\ Ryazan State Radio Engineering University named after V.F. Utkin, Ryazan, Russia, assur@bk.ru
}

The work is developed a numerical-analytical approach to modeling thermal processes in a composite anode of microfocus X-ray tubes. At the first stage of modeling, with the help of well-known analytical approximations, the distribution function of volumetric heat sources is built. At the second stage, this function is used when solving a stationary boundary value problem with the Poisson equation by the Finite Elements Method. Using the proposed technique, an adequate assessment of the maximum power of the tube with the transmission type $W$-Be anode was developed.

Keywords: microfocus X-ray tube, composite anode, volumetric heat sources, Poisson equation.

\section{Introduction}

The use of microfocus tubes vs macrofocus ones in X-ray installations provides a number of indisputable advantages: equipment compactness, decreasing by the radiation load on the personnel and on the adjacent areas of the object under study, the possibility of local research, ability to obtain magnified images, higher image quality with the same radiation doses.

The improvement of microfocus X-ray tubes is under two main directions: 1) an increase in the power of X-ray radiation; 2) reducing the diameter of the focal spot. Both directions are associated with the solution of the dissipation problem by the anode of the microfocus tube of the heat output. The power of the X-ray tube and the diameter of the focus stain are mainly limited by the limit thermal power allocated on the anode. An increase in the specified limit will allow to expand the diagnostic capabilities of X-ray equipment.

Despite the fact that in recent years, $\mathrm{X}$-ray sources have appeared with the anodes of original structures that can dispel significant thermal capacities [1 - 3], most of the commercial and scientific samples of the tubes use inexpensive classic solid composite anodes. Many questions relating to the optimal parameters of such anodes are still open. To obtain an adequate estimate of the capacity of the power allocated in a flat composite anode, it is necessary to clarify the existing models of heat propagation in the respective solid structures.

\section{Analytical approximation of the heat generation area}

Let us to consider the problem of determining the thermal mode of operation of the anode of the microfocus tube of the transmission type. The classic anode of the tube is a film structure in which the target material is applied to the substrate. Electrons, bombarding target, penetrate deep into the material, generating $\mathrm{X}$-ray radiation due to the loss of kinetic energy during their braking. The scattering of electron energy occurs in the interaction area, the dimensions and the shape of which are determined by the accelerating voltage on the anode $U$, the diameter of the focal spot $d_{\mathrm{b}}$ and the atomic number $z$ of the target material [4].

From the point of view of the heat propagation problem, the domain of interaction of the electron beam with the target is an internal heat source, the volumetric power of which is determined by the spatial distribution of energy losses by electrons when they are moved in a solid [5]. In the case of the internal heat sources, the stationary temperature field $T(x, y, z)$ is the solution of the Poisson equation:

$$
\frac{\partial^{2} T}{\partial x^{2}}+\frac{\partial^{2} T}{\partial y^{2}}+\frac{\partial^{2} T}{\partial z^{2}}=-\frac{q_{v}(x, y, z)}{\lambda(T)}
$$

where $q_{v}(x, y, z)$ is the spatial distribution of the volumetric power of the internal heat source, $\lambda(T)$ is the thermal conductivity coefficient depending on temperature. 
One of the main factors affecting the maximum temperature in the target is the size and shape of the heat release region, which is determined by the dependence $q_{v}(x, y, z)$. For practical purposes, the interaction region is approximated by the hemisphere model [4] or, as suggested in this work, by the truncated sphere model shown in Fig. 1.

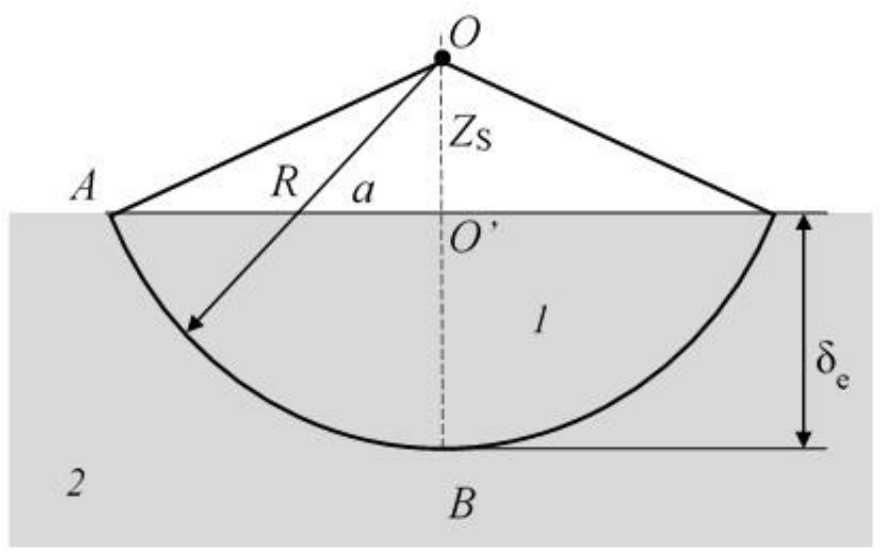

Fig. 1.Approximation of the region of interaction of electrons with the target material by a truncated sphere: 1 - region of interaction; 2 - target.

The radius $\mathrm{R}$ and the value of the displacement of the center of the sphere $Z_{S}$ are found from the triangle $\Delta \mathrm{OO}^{\prime} \mathrm{A}$ by the simplest algebraic calculations

$$
\begin{gathered}
Z_{\mathrm{S}}=\frac{\delta_{\mathrm{e}}}{2}\left(\left(\frac{a}{\delta_{\mathrm{e}}}\right)^{2}-1\right), \\
R=\frac{\delta_{\mathrm{e}}}{2}\left(1+\left(\frac{a}{\delta_{\mathrm{e}}}\right)^{2}\right),
\end{gathered}
$$

where $\delta_{e}$ is the extrapolated depth of path of electrons in a solid, $a$ is the radius of the region of electrons propagation in the near-surface layer of the target, equal to $a=\delta_{\mathrm{e}}+0.5 d_{\mathrm{b}}$.

The well-known semiempirical relations [6] can be used to determine the parameter $\delta_{e}$. The most commonly used in applied research is the formula for determining $\delta_{e}$ in the Cosslett-Thomas model

$$
\delta_{\mathrm{e}}=\frac{0.074 U^{1.55}}{\rho}[\mu \mathrm{m}],
$$

where $U[\mathrm{kV}]$ is the accelerating voltage at the anode, and $\rho\left[\mathrm{g} / \mathrm{cm}^{3}\right]$ is the density of the target material.

The distribution of volumetric heat sources $q_{v}(x, y, z)$ will be determined by the normalized distribution function of electrons energy losses in the interaction region. Approximations of this function are known in one-dimensional and axisymmetric formulations of the problem. In the axisymmetric formulation of the problem, a normalized function of energy losses with a Gaussian distribution is traditionally used to determine volumetric heat sources, which has the form [7]:

$$
q_{\mathrm{v}}(r, z)=A\left(\exp \left(-\left[\frac{r^{2}}{a_{1}{ }^{2}}+\frac{\left(z-z_{\mathrm{ms}}\right)^{2}}{z_{\mathrm{ms}}{ }^{2}}\right]\right)+B \exp \left(-\left[\frac{r^{2}}{a_{2}{ }^{2}}+\frac{\left(z-z_{\mathrm{ss}}\right)^{2}}{z_{\mathrm{ss}}{ }^{2}}\right]\right)\right),
$$


where $A=\frac{0.2(1-\eta) P}{a_{1}^{2} z_{\mathrm{ms}}\left(1-\eta+\eta \frac{z_{\mathrm{ss}}}{z_{\mathrm{ms}}}\right)}, \quad B=\frac{\eta a_{1}^{2}}{(1-\eta) a_{2}^{2}}, \eta$ is the reflection coefficient of primary electrons, parameters $a_{1}{ }^{2}=z_{\mathrm{ms}}{ }^{2}+0.72 d_{\mathrm{b}}{ }^{2}$ и $a_{2}{ }^{2}=0.25 z_{\mathrm{ss}}{ }^{2}+0.72 d_{\mathrm{b}}{ }^{2}, z_{\mathrm{ms}}$ and $\mathrm{z}_{\mathrm{ss}}$ are the depths of maximum electron energy losses for the primary beam and reflected electrons, numerically equal to $1 / 3$ and $1 / 6$ of the extrapolated path depth $\delta_{e}$.

\section{Estimation of the approximation accuracy}

Let us estimate the accuracy of approximation (5) and the possibility of using it in our problem. Full power $P$ supplied to the target is dissipated in the volume of the interaction region $V_{I R}$ (Fig. 1) [8].In this case, the heat release volumetric power can be found as

$$
q_{\mathrm{v}}=\frac{P}{V_{\mathrm{IR}}}=\frac{6 P}{\pi \delta_{\mathrm{e}}\left[3\left(\delta_{\mathrm{e}}+0.5 d_{\mathrm{b}}\right)^{2}+\delta_{\mathrm{e}}^{2}\right]}
$$

Obviously, the average value of the volumetric power for distribution (5) must coincide with $q_{v}$ from (6).The average value of the volumetric power for the distribution $q_{v}(r, z)$ can be found as

$$
\bar{q}_{v}=\int_{r_{1}}^{r_{2}} \int_{z_{1}}^{z_{2}} \frac{q_{\mathrm{v}}(r, z) \partial z \partial r}{\left(r_{2}-r_{1}\right)\left(z_{2}-z_{1}\right)}
$$

For a tungsten $\mathrm{W}$ target and the parameters of an electron beam $U=200 \mathrm{kV}, P=20 \mathrm{~W}, d_{b}=20 \mu \mathrm{m}$, the value of the volumetric power $q_{v}$ is $1.4 \mathrm{~mW} / \mu^{3}$, and the value of the average volumetric power is equal to $\bar{q}_{v}=1.9 \mathrm{~mW} / \mu \mathrm{m}^{3}$, which indicates the correctness of formula (5)and applicability it for our task.In confirmation of the above, Fig.2 shows the spatial distribution of $q_{v}(r, z)$ in the interaction region for the purpose of comparison it with the value $q_{v}=1.4 \mathrm{~mW} / \mu \mathrm{m}^{3}$.

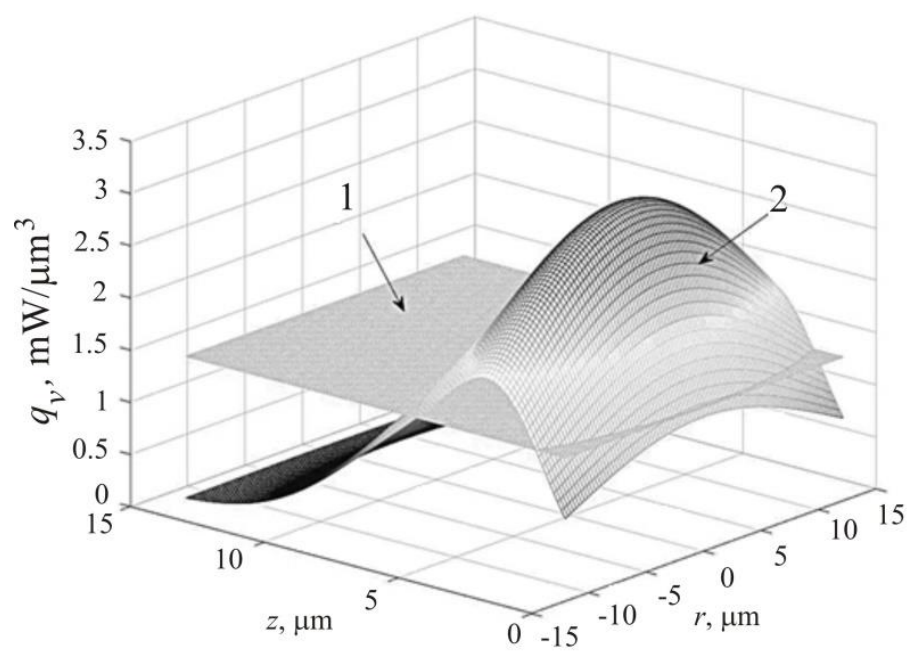

Fig.2. Spatial distribution of volumetric heat sources in the interaction region:

$$
1-q_{v}=1.4 \mathrm{~mW} / \mu \mathrm{m}^{3}, 2-q_{v}(r, z) \text {. }
$$

From the analysis of the data in Fig.2, it can be concluded that the value $q_{v}=1.4 \mathrm{~mW} / \mu \mathrm{m}^{3}$ really adequately reflects the average value of the distribution $q_{v}(r, z)$. Thus, we conclude that relation (5) is 
applicable to the solution of stationary thermal problems for transmission type anodes of the of microfocus $\mathrm{X}$-ray tubes.

\section{Numerical modeling of the heat transfer process in a flat composite anode with an analytically determined heat release region}

Let us consider the problem of determining the thermal mode of operation of the transmission type W$\mathrm{Be}$ anode of a microfocus X-ray tube, taking into account the approximation of the interaction region by a truncated sphere. In view of the complexity of the analytical solution of the heat problem with equation (1), we will use numerical methods. The COMSOL Multiphysics numerical simulation environment allows you to simulate complex thermal problems taking into account all the processes of heat transfer from the solid surface (convection, radiation). The modeling process includes several stages: creation of the geometry of the considered area; selection of materials; setting up the physical interface of heat transfer, namely, setting the initial and boundary conditions, setting the heat source; building a mesh of finite elements; postprocessing of the obtained results.

Fig. 3 shows the computational model of the object to the COMSOL environment.

In modeling, we assume that the layer thickness $\mathrm{W}$ equaled to $\delta_{e}$, is calculated by the Cosslett-Thomas formula (4), and the thickness of the Be layer is $100 \mu \mathrm{m}$. The composite anode diameter is $10 \mathrm{~mm}$. On the outer boundary, we set the condition of natural convection, which is characterized by the heat transfer coefficient $\alpha$. Changing the voltage $U$ at a constant power $P$, we can obtain the dependence $T_{\max }(U)$ for $\mathrm{W}$ and Be at different values of the diameter $d_{b}$. In this case, the beam current lis not taken into account in the simulation.

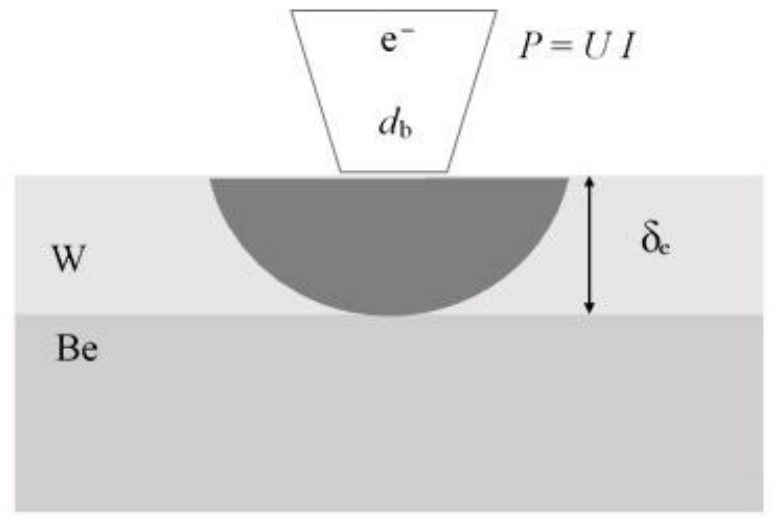

Fig.3. Computational model of heat propagation in ananode of the transmission type.

The purpose of the simulation is to determine the maximum power $P_{\max }$ supplied to the target, since the value of $P_{\max }$ is limited by the temperature value at which the destruction of the anode materials occurs. The simulation results are shown in Fig.4.



a)

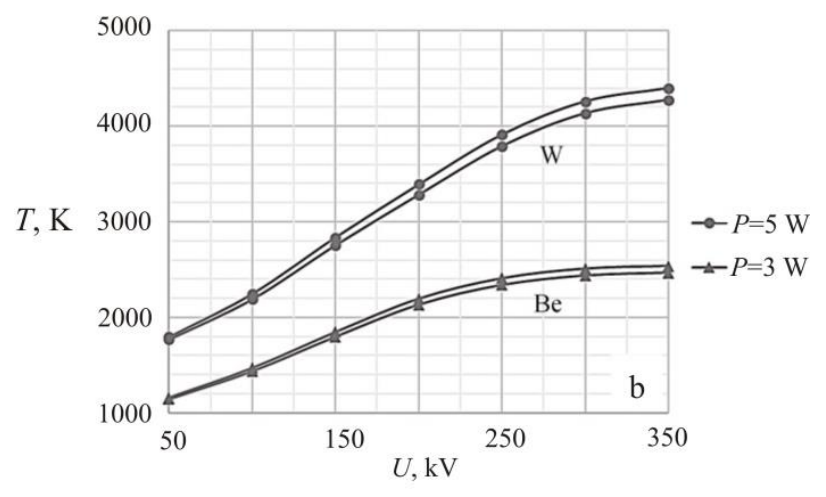

b)

Fig.4. Dependence of the maximum temperature of the materials of the $\mathrm{W}$-Be transmission anode on the voltage $U$ at a fixed power $P$ : a - the size of the focal spot $d_{b}=10 \mu \mathrm{m} ; \mathrm{b}$ - focal spot size $d_{b}=20 \mu \mathrm{m}$ 
Based on the simulation results, it can be concluded that the value of $P_{\max }$ is about $3 \mathrm{~W}$, both for $d_{b}=10$ $\mu \mathrm{m}$ and $d_{b}=20 \mu \mathrm{m}$. In this case, the value of the maximum input power is mainly limited by the temperature of the beryllium substrate; moreover, insignificant changes in $P$ lead to a strong increase in temperature. Noteworthy is the presence of a maximum in the curves, which arises as a result of the fact that with increasing $U$, the temperature difference $\Delta T$ increases due to an increase in the effective thickness $\mathrm{W}$ of the target. The factor of increasing the volume of the interaction region begins to slightly prevail at voltages of the order of $350 \mathrm{kV}$, i.e. at low voltages, it can be ignored.

\section{Conclusion}

Thus, based on the results of the work, a conclusion can be made that taking into account the influence of the distribution $q_{v}(r, z)$ makes it possible to carry out a comprehensive numerical study of the thermal mode of operation of the transmission type anode of microfocus tubes in order to establish the relationship between the main parameters of the anode (accelerating voltage $U$, current $I$, focal spot diameter $d_{b}$ and atomic number $Z$ ) with the power $P$. The established relationships are the theoretical basis for achieving the limiting powers dissipated by solid anodes of microfocus X-ray tubes.

\section{Acknowledgments}

The research was funded by Russian Foundation for Basic Research (RFBR), project number 19-38-90231.

\section{REFERENCES}

1 Hemberg O., Otendal M., Hertz H. M. Liquid-metal-jet anode electron-impact X-ray source. Applied Physics Letters. 2003, Vol. 83, No. 7, pp. 1483-1485.

2 Trubitsyn A.A., Grachev E.Yu., Kozlov E.A. High-power x-ray tube for micro computer tomography. Journal of Physics: Conf. Series. 2019, Vol. 1164, pp. 012022-1 - 012022.

3 Trubitsyn A.A., GrachevE.Yu., Kozlov E.A., Shugayeva T.Zh. Development of High Power Microfocus Tube. Eurasian Physical Technical Journal. 2021, Vol.18, No. 1(35), pp. 43-50.

4 Pozdeeva T.Yu., Smetkin A.A.Monte Carlo simulation of the interaction of an electron beam with matter.Mashinostroyeniye.2017, No. 2, pp. 7-21. [in Russian]

5 Aamir Ihsan, Sung Hwan Heo, Sung Oh Cho. Optimization of X-ray target parameters for a high-brightness microfocus X-ray tube. Nucl.Instr. and Meth.in Phys. Res. B. 2007, Vol. 264, pp. 371 - 377.

6 Lukyanov F.A., Rau E.I., Sennov R.A. Depth of path of primary electrons, spreading of an electron beam and spatial resolution in electron probe studies. Izvestia RAN. Physical series. 2009, Vol. 73, No. 4, pp. 463 - 472 . [in Russian]

7 Amrastanov A.N., Ginzgeimer S.A., Stepovich M.A., Filippov M.N. On one possibility of mathematical modeling of the thermal effect of a sharply focused electron beam on a homogeneous semiconductor. Izvestia RAN. Physical series. 2016, Vol. 80, No. 10, pp.1448-1452. [in Russian]

8 Kozlov E., Trubitsyn A., Fefelov A., Kirushin D. "Computer Simulation of Influence the Interaction Region Electrons at the Maximum Temperature in the Target". Proc. of the 9th Mediterranean Conference on Embedded Computing (MECO 2020), Budva, Montenegro, June 2020. Article number 9134114. 